\title{
Utilização de métodos de previsão da velocidade de ocorrência de aquaplanagem para verificação da segurança em rodovias de alto padrão
}

\author{
Henrique Foster de Oliveira ${ }^{1}$, Felipe Issa Kabbach Jr. ${ }^{2}$, Carlos Yukio Suzuki ${ }^{3}$ \\ ${ }^{1}$ Universidade de São Paulo, Brasil, henrique.foster@usp.br \\ 2Universidade de São Paulo, Brasil, fkabbachjr@planservi.com.br \\ 3Universidade de São Paulo, Brasil, suzuki@planservi.com.br
}

\section{Recebido:}

16 de junho de 2018

Aceito para publicação:

17 de setembro de 2018

Publicado:

31 de dezembro de 2019

Editor de área:

Flávio Cunto

\section{Palavras-chaves:}

Aquaplanagem,

Segurança Viária,

Interface pneu-pavimento.

\section{Keywords:}

Aquaplaning,

Road safety,

Tire-pavement interface.

\begin{abstract}
RESUMO
Este trabalho apresenta de forma resumida diferentes métodos existentes de previsão da velocidade de ocorrência de aquaplanagem para demonstrar o risco associado a este fenômeno e a necessidade de complementação dos critérios e recomendações de projeto utilizados no Brasil, de maneira que seja considerada a probabilidade de ocorrência de tal fenômeno em determinadas situações. Para tanto, é apresentada a análise de um trecho hipotético que combina diversas características típicas de rodovias de alto padrão, localizado na Região Metropolitana de São Paulo.
\end{abstract}

\begin{abstract}
This work presents a summary of existing methods to predict hydroplaning speed limits and it highlights the risks associated with this phenomenon. Based on the assessment of the probability of occurrence of this phenomenon in different scenarios, the work identifies the need for the development of complementary criteria and recommendations for projects in Brazil. A case study is undertaken to assess the proposed methodologies on a hypothetical highway section that combines typical high standards highway conditions and located in the Metropolitan Region of São Paulo.
\end{abstract}

DOI:10.14295/transportes.v27i4.1735 OPEN 2 ACcEss

\section{INTRODUÇÃO}

A aquaplanagem é um fenômeno decorrente da separação entre o pneu e o pavimento pela presença de uma película de água entre eles, resultando em uma ausência de aderência e possivelmente na perda de controle do veículo.

Sua principal ocorrência em rodovias é conhecida como aquaplanagem dinâmica e surge a partir da dificuldade em que a água seja expelida da área de contato com o pavimento devido a elevadas velocidades radiais do pneu (BROWNE, 1975).

Ainda que seja um fenômeno razoavelmente reconhecido e que esteja relacionado a $17 \%$ de todas as colisões e a $23 \%$ das colisões em condições de pavimento úmido na Alemanha (HERRMANN. 2008l. é raro encontrar alguma referência à aquaplanagem em manuais de projeto de rodovias. 
Em anos recentes tal tema tem sido foco de maior interesse acadêmico possivelmente pelo aumento na velocidade regulamentar em rodovias de boas características técnicas, pelos alargamentos do leito carroçável de rodovias e de uma expectativa do impacto advindo das mudanças climáticas.

Assim, esse artigo tem por objetivo apresentar e comparar os métodos existentes de verificação de ocorrência da aquaplanagem, utilizando tais métodos na identificação de trechos rodoviários favoráveis à ocorrência deste fenômeno. Espera-se que os resultados obtidos permitam a identificação de trechos rodoviários para a aplicação de medidas mitigadoras de tal fenômeno.

\section{MÉTODOS DE PREVISÃO}

A aquaplanagem dinâmica ocorre quando a lâmina de fluido presente entre o pavimento e o pneu não consegue ser expelida da interface pneu-pavimento com velocidade suficiente, reduzindo consequentemente a área de contato e eliminando a aderência que garante a estabilidade e controle do veículo, como ilustrado na Figura 1.

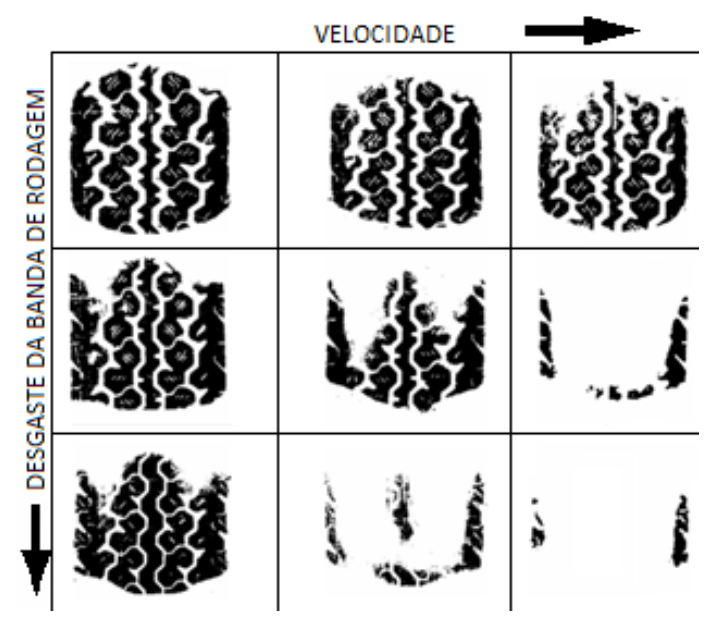

Figura 1. Variação da área de contato na ocorrência de aquaplanagem dinâmica, adaptado de Gothié (2005)

Diversos fatores influenciam a ocorrência da aquaplanagem, os quais podem ser divididos em 4 grupos, conforme apresentado na Figura 2.

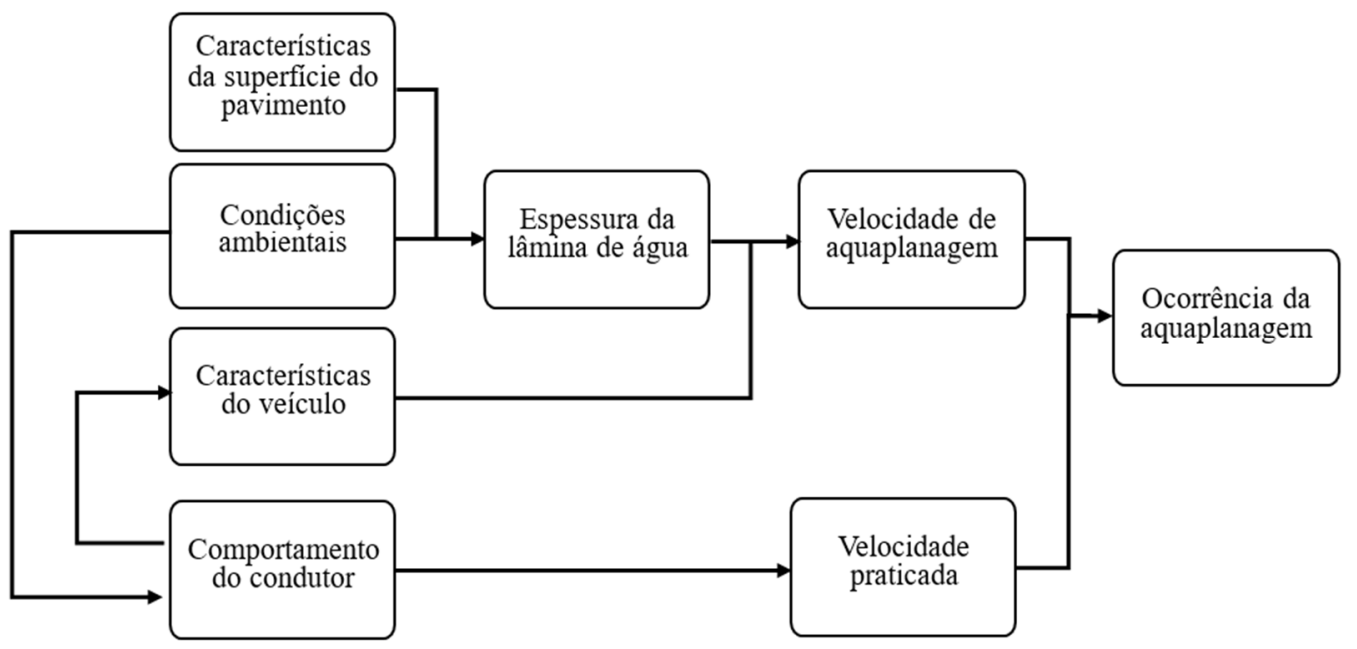

Figura 2. Fluxograma de ocorrência da aquaplanagem 
Salienta-se que a velocidade praticada pelos condutores é dependente, dentre outros fatores, das condições ambientais, especialmente da intensidade de chuva. E há um consenso de que intensidades de precipitação maiores implicam em maiores reduções na velocidade praticada. Contudo, ainda não há um consenso sobre a magnitude dos valores destas reduções. Assim, apesar das intensidades de chuva consideradas neste trabalho serem classificadas como chuvas intensas de acordo com o Highway Capacity Manual (2010), as condições de visibilidade resultantes são suficientes para a circulação à velocidade de projeto considerada (IVEY, LEHTIPUU e BUTTON, 1975) e portanto não serão consideradas as reduções de velocidade na identificação de trechos críticos.

Os modelos de verificação do risco de aquaplanagem considerados no presente trabalho avaliam apenas a ocorrência de aquaplanagem dinâmica nas rodovias, sendo que os mesmos podem ser divididos em dois grupos: os que permitem a previsão da espessura de lâmina de água no pavimento e aqueles que permitem a previsão da velocidade de ocorrência da aquaplanagem.

Na descrição resumida dos modelos apresentados mais adiante, os nomes e unidades das variáveis foram padronizados de acordo com o indicado na Tabela 1:

Tabela 1 - Padronização das unidades das variáveis

\begin{tabular}{lll}
\hline Variável & Descrição & Unidade \\
\hline$H_{F}$ & Espessura da lâmina de água acima da macrotextura do pavimento & $\mathrm{mm}$ \\
$T M P$ & Altura média da macrotextura do pavimento & $\mathrm{mm}$ \\
$L_{F}$ & Comprimento da linha de água & $\mathrm{m}$ \\
$I$ & Intensidade pluviométrica & $\mathrm{mm} / \mathrm{h}$ \\
$S_{F}$ & Declividade da linha de água & $\mathrm{m} / \mathrm{m}$ \\
$n$ & Coeficiente de rugosidade de Manning & - \\
$V_{H}$ & Velocidade de ocorrência da aquaplanagem & $\mathrm{km} / \mathrm{h}$ \\
$F A R$ & Relação largura/extensão da área de contato pneu-pavimento & - \\
$P_{P}$ & Pressão do pneu & $\mathrm{kPa}$ \\
$S D$ & Redução relativa da rotação da roda no início da aquaplanagem & $\%$ \\
$S_{P}$ & Profundidade de sulco da banda de rodagem & $\mathrm{mm}$ \\
$\varphi$ & Fração da área de contato dos pneus que é composta por sulcos & $\%$ \\
$P_{C}$ & Carga por roda & $\mathrm{kN}$ \\
$P_{C, \text { perm }}$ & Carga por roda máxima permitida & $\mathrm{kN}$ \\
$P_{L}$ & Largura da área de contato do Pneu & $\mathrm{mm}$ \\
\hline
\end{tabular}

Os modelos mais antigos mencionados em várias publicações são os elaborados pela National Aeronautics and Space Admnistration (HORNE e DREHER, 1963) (HORNE, YAGER e TAYLOR, 1968), modelos esses desenvolvidos com foco na operação de aeronaves. Esses modelos não consideravam a existência de sulcos na banda de rodagem dos pneus e foram validados para espessuras de lâmina de água maiores do que $7 \mathrm{~mm}$. Contudo, posteriormente, foram adaptados para veículos rodoviários de carga e dimensões de pneus típicas destes veículos.

O desenvolvimento mais recente deste modelo, apresentado na equação (1), desenvolvido por Horne (1986), é o único, dentre os modelos estudados, que não utiliza uma variável para a espessura da lâmina de água.

$$
V_{H}=83,363812-(27,600181 \times F A R)+\left(0,168059 \times P_{P}\right)
$$

Nos estudos do Road Research Laboratory (ROSS e RUSSAM, 1968), que objetivaram a avaliação da espessura da lâmina de água na superfície do pavimento sem associação direta com 
estudos de ocorrência de aquaplanagem, os modelos propostos foram utilizados posteriormente em conjunto com modelos de outros autores para a previsão do risco de aquaplanagem. Dos modelos criados pelo RRL, destaca-se o apresentado na equação (2), por resultar em uma lâmina d'água acima das asperezas do pavimento.

$$
H_{F}=0,046\left[\frac{\left(L_{F} \times I\right)^{0,5}}{S_{F}^{0,2}}\right]
$$

Estes estudos acabaram sendo menos mencionados após a divulgação do modelo proposto por Gallaway (1979), que utilizou dados combinados de pista de teste e ensaios de laboratório para avaliar o risco de aquaplanagem em rodovias. Os modelos foram propostos considerando as características de veículos de passeio, em contraste com os modelos anteriores.

0 modelo de Gallaway foi dividido nas equações (3) a (5), sendo que a equação (4), que já havia sido proposta por Agrawal (1977), é válida somente para espessuras de lâmina de água menores do que $2,4 \mathrm{~mm}$.

$$
\begin{gathered}
H_{F}=0,01485\left[T M P^{0,11} \times L_{F}^{0,43} \times I^{0,59} \times S_{F}^{-0,42}\right]-T M P \\
V_{H}=96,9 \times H_{F}{ }^{-0,259} \\
V_{H}=0,9143 \times S D^{0,04} \times P_{P}^{0,3} \times\left(S_{P}+0,794\right)^{0,06} \times \text { máximo } \\
\left(\begin{array}{c}
\frac{12,369}{H_{F}^{0,06}}+3,507 \\
\left.\frac{22,351}{H_{F}^{0,06}}-4,97\right) \times T M P^{0,14}
\end{array}\right.
\end{gathered}
$$

Em uma publicação do National Highway Cooperative Research Board (1998) foi apresentado um software para Ms-DOS designado por PAVDRN; neste software o modelo de Gallaway foi adaptado, com adoção de valores fixos para algumas variáveis. Neste estudo foi considerado que a equação (3), referente à espessura de lâmina d'água, seria inadequada por possuir validade limitada, tendo sido proposta a equação (6), baseada nas soluções de onda cinemática para o caso de saída de fluxo constante.

$$
H_{F}=\left(\frac{n \times L_{F} \times I}{36,1 \times S_{F}{ }^{0,5}}\right)^{0,6}-T M P
$$

Outra linha de pesquisa que foi desenvolvida é baseada em métodos de simulação computacional das condições de fluxo de água entre o pneu e o pavimento, resultando na velocidade de ocorrência de aquaplanagem. Nessa linha destacam-se os trabalhos de Ong e Fwa (2007) (2008), que foram adaptados por Gunaratne (2012) na equação (7).

$$
V_{H}=\left(P_{C} \times 1000\right)^{0,2} \times P_{P}^{0,5}\left(\frac{0,82}{H_{F}^{0,06}}+0,49\right)
$$

A principal deficiência desse modelo é a sua validação a partir do modelo da NASA (HORNE, YAGER e IVEY, 1986), que foi calibrado para a aquaplanagem de aeronaves.

Para a velocidade de ocorrência da aquaplanagem há o método proposto na Alemanha por Gengenbach (1967), o qual considera o uso de pneus sem ranhuras e que foi posteriormente adaptado por Höcker (1971), permitindo a sua aplicação para as condições atuais de operação rodoviária. Tal modelo é caracterizado na equação (8). 


$$
V_{H}=5080 \times \sqrt{\frac{P_{C}}{P_{L} \times\left[H_{F}-\varphi \times\left(S_{P}-0,8\right)\right] \times\left[46 \times\left(\frac{P_{C}}{P_{c, p e r m}}\right)^{-1,342}\right]}}
$$

Por último, temos o método de avaliação da espessura da lâmina de água sobre o pavimento proposto por Herrmann (2008), o qual é baseado na solução de onda cinemática de saída variável e calibrado a partir de dados de campo para utilização de diversos ensaios de macrotextura do pavimento. Na equação (9) é apresentada a variação da equação calibrada para uso do ensaio de mancha de areia como medida da macrotextura do pavimento.

$$
H_{F}=0,26334 \times\left(L_{F} \times \frac{I}{60}\right)^{0,4158} \times S_{F}^{-0,3314} \times T M P^{0,4177}-T M P
$$

Analisando as equações apresentadas e as variáveis que foram utilizadas em cada caso, resumidas na Tabela 2, podem ser destacados os seguintes fatos:

- O modelo da NASA é o único a não considerar a espessura da lâmina de água no cálculo da velocidade de ocorrência da aquaplanagem;

- O modelo de velocidade de Gallaway considera apenas a espessura da lâmina d'agua para as espessuras usuais dos casos estudados;

- Dentre as variáveis consideradas nas equações de previsão da espessura da lâmina d'água, a que apresenta maior impacto no resultado é a intensidade de chuva, seguida pelo comprimento da linha de fluxo d'água.

Tabela 2 - Resumo das variáveis utilizadas em cada equação

\begin{tabular}{lllllllll}
\hline Espessura da Lâmina de água & & & & & & & \\
\hline & $T M P$ & $L_{F}$ & $\mathrm{I}$ & $S_{F}$ & $n$ & & \\
\hline RRL (equação 2) & & $\mathrm{X}$ & $\mathrm{X}$ & $\mathrm{X}$ & & & \\
\hline Gallaway (equação 3) & $\mathrm{X}$ & $\mathrm{X}$ & $\mathrm{X}$ & $\mathrm{X}$ & & & \\
\hline PAVDRN (equação 6) & $\mathrm{X}$ & $\mathrm{X}$ & $\mathrm{X}$ & $\mathrm{X}$ & $\mathrm{X}$ & & & \\
\hline Herrmann (equação 9) & $\mathrm{X}$ & $\mathrm{X}$ & $\mathrm{X}$ & $\mathrm{X}$ & & & & \\
\hline Velocidade de ocorrência da aquaplanagem & & & & & & \\
\hline \multicolumn{1}{l}{$H_{F}$} & $T M P$ & $F A R$ & $P_{P}$ & $S D$ & $S_{P}$ & $P_{C}$ & $P_{L}$ \\
\hline NASA (equação 1) & & & $\mathrm{X}$ & $\mathrm{X}$ & & & & \\
\hline Gallaway (equações 4 e 5) & $\mathrm{X}$ & $\mathrm{X}$ & & $\mathrm{X}$ & $\mathrm{X}$ & $\mathrm{X}$ & & \\
\hline Gunaratne (equação 7) & $\mathrm{X}$ & & & $\mathrm{X}$ & & & $\mathrm{X}$ & \\
\hline Gengenbach (equação 8) & $\mathrm{X}$ & & & & & $\mathrm{X}$ & $\mathrm{X}$ & $\mathrm{X}$ \\
\hline
\end{tabular}

Dentre os métodos de previsão da espessura da lâmina de água no pavimento apresentados, os propostos por Herrmann e pelo NHCRB são baseados em conceitos teóricos e calibrados com dados de ensaios, enquanto os outros são baseados em modelos de regressão utilizando dados empíricos, sendo que todos consideram a espessura de lâmina de água acima da macrotextura do pavimento.

Os métodos para determinação da velocidade de ocorrência de aquaplanagem são empíricos, com os modelos propostos por Gallaway, Gengenbach e Horne sendo calibrados com dados experimentais e o proposto por Gunaratne sendo calibrado por modelos computacionais. E observa-se que quase todos os modelos apresentados foram desenvolvidos há mais de 40 anos, sendo que tal fato se deve a uma tendência de trabalhos atuais possuírem enfoque na aplicação destes modelos em casos específicos. 
Algumas observações sobre o desempenho dos modelos de previsão da espessura da lâmina de água apresentados são:

- Pavimentos com valores maiores de macrotextura implicam em menores espessuras de lâmina de água, com exceções ocorrendo para o método do RRL (pois este não considera esta variável) e para o modelo de Herrmann em que o efeito é reduzido com o aumento das intensidades de chuva;

- $\quad$ coeficiente de Manning, utilizado no método do PAVDRN, não é influenciado pela macrotextura, sendo dependente apenas da intensidade de chuva, do comprimento da linha de fluxo e da temperatura d'água;

- A sensibilidade das equações em relação ao comprimento da linha de fluxo de água e intensidade de precipitação é semelhante entre os 4 métodos avaliados; tal fato pode ser observado pela proximidade dos valores dos expoentes aplicados a estas variáveis;

- O impacto causado pela variação da declividade média da linha de fluxo de água diminui consideravelmente para valores maiores do que $2 \%$, indicando que a existência de linhas de fluxo de água com declividades menores que esta são críticas à operação rodoviária;

- As espessuras previstas pelo modelo do RRL têm valores geralmente superiores a 1,5 mm em condições consideradas típicas, possivelmente em decorrência da restrição durante a coleta de dados do experimento original.

E em relação às equações de velocidade de ocorrência da aquaplanagem observa-se que:

- 0 modelo desenvolvido por Gunaratne possui a menor variabilidade dentre os 4 apresentados, com velocidades previstas para ocorrência de aquaplanagem entre 75 e 100 $\mathrm{km} / \mathrm{h}$;

- Os resultados do modelo da NASA dependem apenas das características do pneu; assim, este modelo não é recomendável na identificação de trechos críticos de rodovias;

- As equações de Gallaway sugerem que seriam necessárias condições extremas para ocorrência de aquaplanagem em velocidades inferiores a $70 \mathrm{~km} / \mathrm{h}$, em desacordo com o modelo de Gengenbach;

- A equação de Gengenbach é a mais sensível aos valores da profundidade dos sulcos da banda de rodagem, possivelmente por considerar esta variável como um redutor da espessura da lâmina de água.

\section{ESTUDO DE CASO}

Para caracterização do procedimento de análise de possibilidade de ocorrência do fenômeno da aquaplanagem, foi elaborada a avaliação de um cenário, composto por um traçado típico de uma rodovia de classe 0, situado na Região Metropolitana de São Paulo, cuja velocidade de projeto é de $110 \mathrm{~km} / \mathrm{h}$ e cuja seção típica é apresentada na figura 2. Na sequência da avaliação foram adicionadas as faixas de tráfego 4 e 5 para realização da análise comparativa entre a situação original e a de pista alargada.

0 traçado objeto de análise possui extensão de $10 \mathrm{~km}$ e foi elaborado de maneira a criar uma variada combinação de declividades horizontais e transversais, representadas na figura 3. Tal traçado é composto por 6 curvas de raios variando entre 900 m e 2000 m, sendo 3 curvas à direita e 3 curvas à esquerda, nas quais, dada a magnitude dos raios adotados, não foram utilizadas curvas de transição.

0 perfil tem rampas com declividade variando de $0,35 \%$ a $4 \%$ e valores de $\mathrm{K}$ (taxa de variação da declividade longitudinal nas curvas verticais) variando de 66 a 170. 


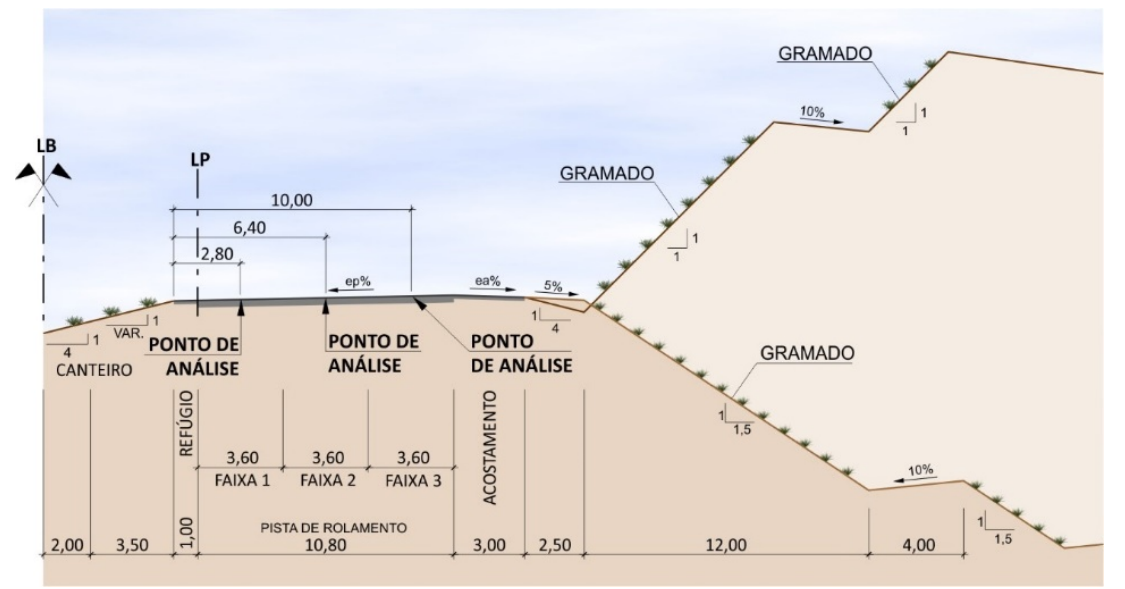

Figura 3. Seção típica de projeto

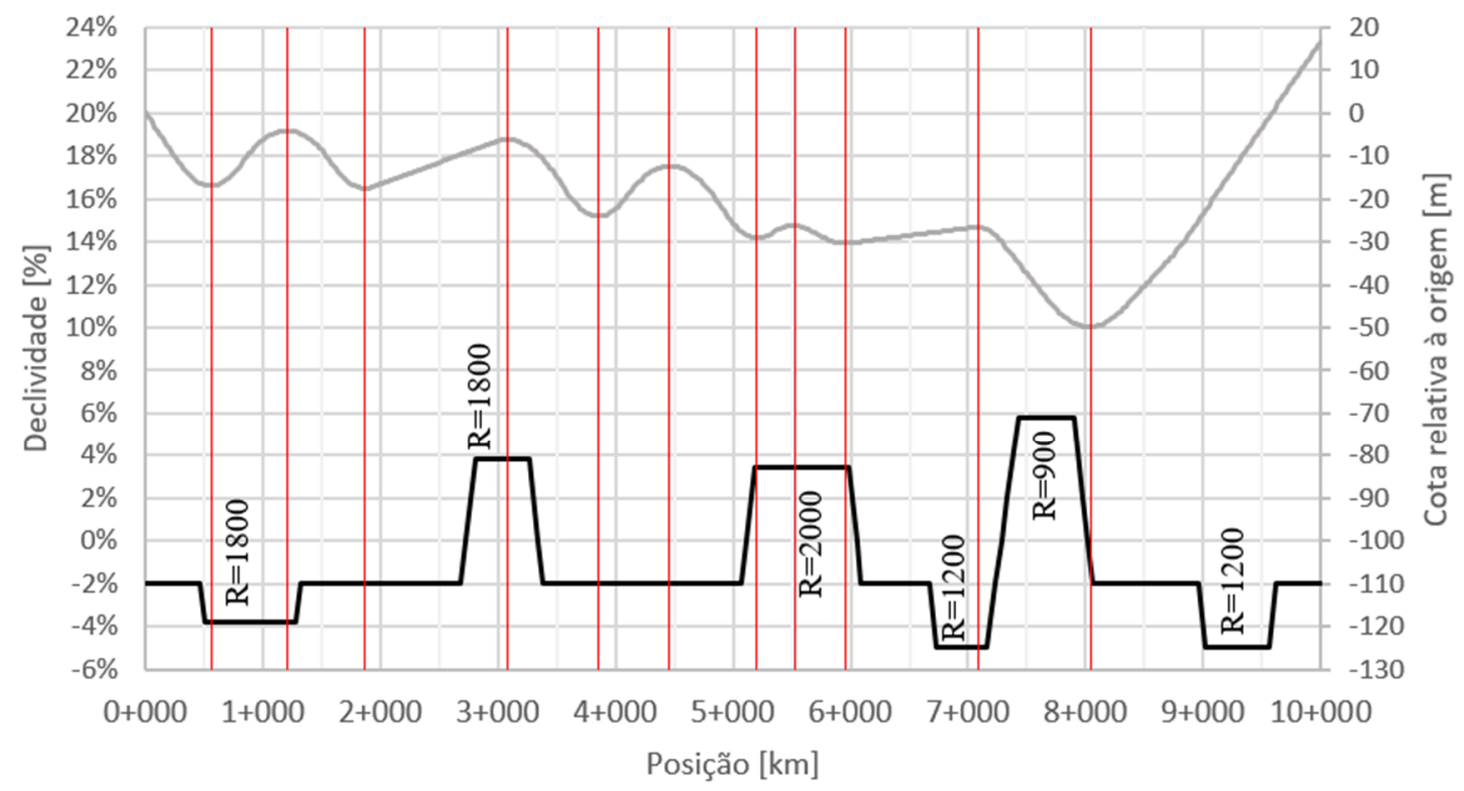

- Superelevação Perfil —— Ponto Alto/Ponto Baixo

Figura 4. Gráfico de superelevação e perfil longitudinal do trecho analisado

Partindo deste traçado hipotético, foram levantadas as informações de declividade transversal e longitudinal para determinação dos comprimentos e declividades das linhas de fluxo de água.

Tais cálculos foram elaborados com auxílio de um algoritmo elaborado com a linguagem “Visual Basic for Applications" em planilha Excel. Com esse algoritmo foi possível obter os valores de comprimento da linha de fluxo do bordo mais alto do pavimento até o ponto de análise, sendo que a declividade foi calculada através do método da área equivalente.

Os dados foram obtidos considerando um ponto inicial de linha de fluxo de água a cada 0,5 $\mathrm{m}$ no bordo do pavimento mais alto, sendo que a superfície do pavimento foi convertida em uma malha de $0,5 \mathrm{~m}$ de espaçamento entre os pontos.

Para as variáveis com maior influência nos modelos, foram adotados 2 valores típicos: um valor médio representativo de todo o espectro de tal variável; e um valor crítico, representativo dos valores mais favoráveis à ocorrência da aquaplanagem. Conforme apresentado a seguir:

- Para a profundidade dos sulcos da banda de rodagem, foi adotado o valor mínimo de 1,6 mm (mínimo permitido para circulação no Brasil de acordo com Congresso Nacional 
(1997)) e médio de 5,5 mm, correspondente ao 50ำ percentil obtido em levantamento nos Estados Unidos (SUBRAMANIAN e THIRIEZ, 2001);

- Para a pressão de enchimento dos pneus foi adotado o valor mínimo de $165 \mathrm{kPa}$ e médio de $210 \mathrm{kPa}$, equivalentes respectivamente aos $90^{\circ}$ e $50^{\circ}$ percentis considerando levantamento realizado nos Estados Unidos (SUBRAMANIAN e THIRIEZ, 2001);

- Para a intensidade de chuva foram adotados como valores máximo $30 \mathrm{~mm} / \mathrm{h}$ e mínimo $10 \mathrm{~mm} / \mathrm{h}$, os quais tem sido superados na Região Metropolitana de São Paulo, respectivamente, durante $5 \mathrm{~h}$ /ano e $35 \mathrm{~h}$ /ano em média (IAG-USP, 2016).

- O valor da relação entre largura e comprimento da área de contato entre o pneu e o pavimento foi calculado a partir dos dados de pressão e largura das rodas, considerando um formato elíptico para a área de contato, tendo resultado em 0,63 quando utilizados os valores críticos e 0,49 quando utilizados os valores médios;

Para as demais variáveis foi adotado um valor médio de acordo com os critérios a seguir:

- A macrotextura do pavimento foi adotada como 0,6 mm, valor médio obtido com os dados de ensaio de mancha de areia fornecidos pela ARTESP para os anos de 2014 a 2017 em diversas rodovias de mesma característica do trecho estudado;

- O valor da largura da área de contato do pneu foi adotado igual a $175 \mathrm{~mm}$, o qual corresponde à média dos valores de modelos disponíveis para veículos de passeio;

- Para a carga por roda foi adotado o valor de $2,5 \mathrm{kN}$, com base na média dos valores de peso bruto de carros de passeio;

- O valor da redução relativa na rotação da roda foi adotado como 10\%, segundo proposto por Gallaway (1979);

- O valor da relação entre a área dos sulcos e a área de contato do pneu com o pavimento foi adotado como igual a de $30 \%$, conforme Herrmann (2008);

- O valor da temperatura da água foi adotado em $21^{\circ} \mathrm{O}$, de acordo com os métodos de previsão de temperatura na superfície do pavimento (ARIAWAN, SUBAGIO e SETIADJI, 2015), utilizando os valores médios de temperatura do ar para a cidade de São Paulo.

A análise do trecho foi elaborada considerando as possíveis combinações de equações, conforme Tabela 3. Para tais combinações foram considerados 2 cenários: o primeiro adota os valores mais críticos para todas as variáveis e o outro adota os valores médios.

Tabela 3 - Combinações possíveis dentre as equações apresentadas

\begin{tabular}{lll}
\hline No da combinação & $\begin{array}{l}\text { Método de previsão da velocidade de ocorrência } \\
\text { da aquaplanagem }\end{array}$ & $\begin{array}{l}\text { Método de previsão da espessura de lâmina } \\
\text { de água }\end{array}$ \\
\hline 1 & Gengenbach (equação 8) & Gallaway (equação 3) \\
\hline 2 & Gengenbach (equação 8) & RRL (equação 2) \\
\hline 3 & Gengenbach (equação 8) & PAVDRN (equação 6) \\
\hline 4 & Gengenbach (equação 8) & Herrmann (equação 9) \\
\hline 5 & Gallaway (equações 4 e 5) & Gallaway (equação 3) \\
\hline 6 & Gallaway (equações 4 e 5) & RRL (equação 2) \\
\hline 7 & Gallaway (equações 4 e 5) & PAVDRN (equação 6) \\
\hline 8 & Gallaway (equações 4 e 5) & Herrmann (equação 9) \\
\hline 10 & Gunaratne (equação 7) & Gallaway (equação 3) \\
\hline 11 & Gunaratne (equação 7) & RRL (equação 2) \\
\hline 12 & Gunaratne (equação 7) & PAVDRN (equação 6) \\
\hline 13 & Gunaratne (equação 7) & Herrmann (equação 9) \\
\hline
\end{tabular}




\section{RESULTADOS E DISCUSSÕES}

Os resultados obtidos foram analisados a partir de gráficos de velocidade de ocorrência da aquaplanagem para cada faixa de tráfego. A título de exemplo são apresentados na Figura 4 os resultados obtidos para a Combinação 8 no cenário que considera os valores críticos, pelo fato de tais resultados possuírem maior variabilidade. Na Figura 4 também é possível evidenciar um fenômeno que se repete em todos os casos analisados; a saber: no entorno dos pontos de transição de superelevação em que esta se aproxima de $0 \%$ existe uma drástica redução na velocidade de ocorrência de aquaplanagem.

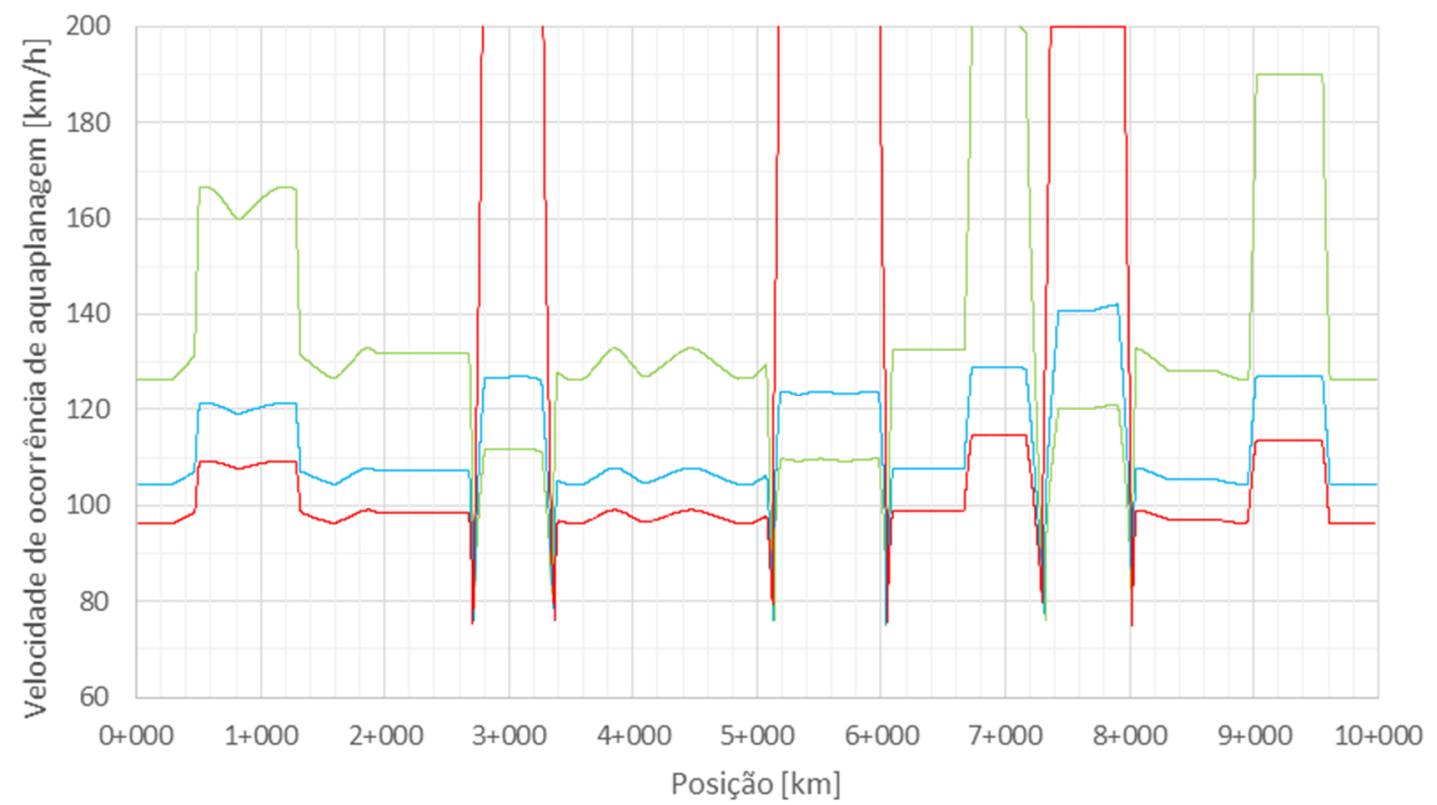

Figura 5. Variação da velocidade de ocorrência da aquaplanagem ao longo da rodovia para a combinação 8 no cenário com valores críticos

A Tabela 4 apresenta as extensões acumuladas em que a velocidade de projeto é maior do que a velocidade de ocorrência de aquaplanagem para cada uma das 13 combinações, separadas por cada faixa de tráfego.

Tabela 4 - Extensão em que a velocidade de ocorrência de aquaplanagem está abaixo da velocidade de projeto 3 faixas de tráfego

\begin{tabular}{lllllll}
\hline \multirow{2}{*}{ Combinação } & \multicolumn{3}{l}{ Cenário com valores críticos } & \multicolumn{3}{l}{ Cenário com valores médios } \\
\cline { 2 - 7 } & Faixa 1 & Faixa 2 & Faixa 3 & Faixa 1 & Faixa 2 & Faixa 3 \\
\hline 1 & 252 & 250 & 255 & 0 & 0 & 0 \\
2 & 6035 & 9974 & 7814 & 32 & 35 & 29 \\
3 & 4904 & 9453 & 7790 & 88 & 96 & 88 \\
4 & 280 & 273 & 3209 & 0 & 0 & 0 \\
5 & 457 & 458 & 5804 & 83 & 90 & 88 \\
6 & 9988 & 9974 & 9831 & 5078 & 9845 & 7783 \\
7 & 8139 & 9974 & 7874 & 781 & 3745 & 6724 \\
8 & 1355 & 5910 & 6704 & 170 & 162 & 164 \\
9 & 8974 & 9974 & 8036 & 505 & 5863 & 5853 \\
10 & 9988 & 9974 & 9960 & 9988 & 9974 & 9960 \\
11 & 9988 & 9974 & 9960 & 8379 & 9974 & 7897 \\
12 & 9988 & 9974 & 9400 & 2505 & 7957 & 7810 \\
13 & 10000 & 10000 & 10000 & 10000 & 10000 & 10000 \\
\hline
\end{tabular}


A partir destes resultados pode-se verificar que, mesmo em um cenário que considera os valores médios, as equações propostas por Gunaratne e NASA resultam em velocidades de ocorrência de aquaplanagem inferiores à velocidade de projeto em praticamente toda a extensão da rodovia. Tal constatação indica um grande conservadorismo destas equações em relação às demais, em decorrência possivelmente da utilização apenas de pneus sem ranhuras em seu desenvolvimento.

Outro resultado observável é que as combinações que utilizam as equações de espessura da lâmina de água do RRL e do PAVDRN resultam em maiores extensões com velocidades abaixo da velocidade de projeto. Sendo que no caso do RRL esses valores são justificados pelo fato do método de medição utilizado na espessura da lâmina de água que não permitia a medição de lâminas com espessura menor do que 1,5 mm. E no caso da equação do PAVDRN essa diferença é justificada pela sensibilidade da equação ao coeficiente de Manning utilizado, coeficiente esse que depende de muitas condições e tem difícil determinação em um ambiente de projeto.

As equações de previsão de espessura da lâmina de água de Gallaway e de Herrmann conduzem a valores resultantes próximos, sendo que tal característica é coerente com a comparação realizada entre o método de Gallaway e a aplicação da modelagem de onda cinemática utilizada por Sheridan (2014)

Portanto, optou-se por descartar as combinações que utilizam essas equações e prosseguir a análise com as combinações 1, 4, 5 e 8. Com o menor número de combinações foram realizadas simulações para 4 e 5 faixas de tráfego, considerando as mesmas características geométricas, cujos resultados são apresentados nas Tabela 5 e 6.

Nota-se uma grande variação nos resultados, principalmente quando alteradas a equação de velocidade de ocorrência de aquaplanagem e o cenário utilizado, sendo necessários mais estudos para correlacionar o cenário e a efetiva ocorrência de acidentes relacionados a este fenômeno. E como esperado, o comprimento de trechos sujeitos à ocorrência de aquaplanagem é proporcional ao número de faixas da rodovia, principalmente nas faixas localizadas à direita da pista (no sentido de circulação do tráfego).

Tabela 5 - Extensão em que a velocidade de ocorrência de aquaplanagem está abaixo da velocidade de projeto considerando valores médios -3 a 5 faixas de tráfego

\begin{tabular}{lllllll}
\hline \multirow{2}{*}{ Combinação } & \multicolumn{5}{l}{ Cenário com valores médios } \\
\cline { 2 - 7 } & Faixa 1 & Faixa 2 & Faixa 3 & Faixa 4 & Faixa 5 \\
\hline 1 & 3 Faixas & 0 & 0 & 0 & - & - \\
1 & 4 Faixas & 0 & 0 & 0 & 0 & - \\
1 & 5 Faixas & 0 & 0 & 0 & 0 & 0 \\
4 & 3 Faixas & 0 & 0 & 0 & - & - \\
4 & 4 Faixas & 0 & 0 & 0 & 0 & - \\
4 & 5 Faixas & 1 & 0 & 0 & 1 & 1 \\
5 & 3 Faixas & 83 & 90 & 88 & - & - \\
5 & 4 Faixas & 120 & 122 & 124 & 117 & - \\
5 & 5 Faixas & 149 & 159 & 156 & 153 & 146 \\
8 & 3 Faixas & 170 & 162 & 164 & - & - \\
8 & 4 Faixas & 227 & 213 & 216 & 224 & - \\
8 & 5 Faixas & 287 & 270 & 268 & 276 & 4593 \\
\hline
\end{tabular}


Tabela 6 - Extensão em que a velocidade de ocorrência de aquaplanagem está abaixo da velocidade de projeto considerando valores críticos -3 a 5 faixas de tráfego

\begin{tabular}{lllllll}
\hline \multirow{2}{*}{ Combinação } & \multicolumn{6}{l}{ Cenário com valores críticos } \\
\cline { 2 - 7 } & Faixa 1 & Faixa 2 & Faixa 3 & Faixa 4 & Faixa 5 \\
\hline 1 & 3 Faixas & 252 & 250 & 255 & - & - \\
1 & 4 Faixas & 330 & 327 & 329 & 5661 & - \\
1 & 5 Faixas & 408 & 405 & 405 & 5734 & 5726 \\
4 & 3 Faixas & 280 & 273 & 3209 & - & - \\
4 & 4 Faixas & 384 & 367 & 3292 & 5731 & - \\
4 & 5 Faixas & 852 & 471 & 3385 & 5813 & 6066 \\
5 & 3 Faixas & 457 & 458 & 5804 & - & - \\
5 & 4 Faixas & 1830 & 600 & 5942 & 6694 & - \\
5 & 5 Faixas & 1879 & 1974 & 6084 & 6832 & 7718 \\
8 & 3 Faixas & 1355 & 5910 & 6704 & - & - \\
8 & 4 Faixas & 1905 & 6904 & 6874 & 7736 & - \\
8 & 5 Faixas & 2400 & 7453 & 7868 & 7907 & 7722 \\
\hline
\end{tabular}

\section{CONCLUSÕES}

As características geométricas da pista do estudo de caso considerado correspondem a condições típicas de rodovias de alto padrão na Região Metropolitana de São Paulo, sendo que os resultados obtidos são representativos do risco de ocorrência de aquaplanagem nesse tipo de rodovia localizado na mesma região.

Tendo em vista estas considerações pode-se afirmar que, nos dois cenários analisados, existe o risco de aquaplanagem em alguns trechos do traçado, o qual está associado principalmente às declividades resultantes nos locais de transição de superelevação.

Também quando considerado o cenário que utiliza valores críticos, nos trechos em tangente existe o risco de ocorrência de aquaplanagem nas faixas de tráfego situadas à direita da pista já a partir da 3aㅡ faixa de tráfego, condição essa que se agrava com a adição de mais faixas.

Apesar do conhecimento deste fenômeno e dos diferentes métodos concebidos para previsão de sua ocorrência, atualmente os manuais de projeto geométrico utilizados no Brasil e no exterior não estabelecem um padrão para a verificação deste risco. Os resultados obtidos no estudo de caso apresentado no presente artigo indicam a conveniência de complementação destes documentos de modo a padronizar os critérios de análise para este tipo de ocorrência.

\section{REFERÊNCIAS}

Agrawall, S. K.; Meyer, W. E.; J.J., H. (1977) Measurement of hydroplaning potential. Pennsylvania Tranportation Institute, Pennsylvania State University, PA, USA: [s.n.].

Ariawan, I. M. A.; Subagio, B. S.; Setiadji, B. H. (2015) Development of asphalt pavement temperature model for tropical climate conditions in West Bali region. The 5th International Conference of Euro Asia Civil Engineering Forum (EACEF-5). [S.l.]: [s.n.]. p. 474-480.

Browne, A. L. (1975) A Mathematical Analysis for Pneumatic Tire Hydroplaning. Surface Texture Versus Skidding: Measurements, Frictional Aspects and Safety Features of Tire-Pavement Interactions, ASTM STP 583, p. 75-94.

Cogresso Nacional. (1997) Código de Trânsito Brasileiro. Brasília: [s.n.].

Gallaway, B. M. et al. (1979) Pavement and geometric design criteria for minimizing Hydroplanning, Report No. FHWARD-79-31. Washington, DC, USA: Federal Highway Administration.

Gengenbach, W. (1967) Das Verhalten von Kraftfahrzeugreifen auf trockener und insbesondere auf nasser Fahrbahn. Karlsruhe: Fakultät für Maschinenbau und Verfahrenstechnik der Universität Karlsruhe.

Gothié, M. (2005) Water influence on skid resistance - Standardization: input of the HERMES programme. International Surface Friction Conference: roads and runways: improving safety through assessment and design. Christchurch, New Zealand : [s.n.]. p. 50.

Gunatne, M. et al. (2012) Hydroplaning on multi lane facilities, Report No. BDK84 977-14. FHWA. University of South Florida, Tampa, FL, p. 130. 
Herrmann, S. R. (2008) Simulationsmodell Zum Wasserabfluss- Und Aquaplaning-Verhalten Auf Fahrbahnoberflächen. Stuttgart: Universität Stuttgart Institut für Straßen- und Verkehrswesen.

Hocker, H. J. (1971) Die Oberflächenentwässerung von Fahrbahnen und ihre Bedeutung für den Straßenentwurf. Aachen: Bundesminister für Verkehr, Abteilung Straßenbau.

Horne, W. B.; Dreher, R. C. (1963) Phenomena of pneumatic tire hydroplaning. Langley Research Center, Langley Station, Washington D.C.: National Aeronautics and Space Administration.

Horne, W. B.; Yager, T. J.; Ivey, D. L. (1986) Recent Studies to Investigate Effects of Tire Footprint Aspect Ratio on Dynamic Hidroplaning Speed. The Tire Pavement Interface, ASTM STP 929, Philadelphia, p. 22-46.

Horne, W. B.; Yager, T. J.; Taylor, G. R. (1968) Review Of Causes And Alleviation Of Low Tire Traction On Wet Runways. Washington, DC: National Aeronautics and Space Administration.

Instituto de astronomia, geofísica e ciências atmosféricas. (2016) Dados horários de precipitação 2000-2015. Universidade de São Paulo. São Paulo, SP.

Ivey, D. L.; Lehtipuu, E. K.; Button, W. (1975) Rainfall and Visibility - The view from behind the wheel. Texas Transportation Institute. College Station, TX, p. 51.

Nhcrp. (1998) Improved surface drainage of pavements (Project 1-29 Final Report). Washington, D.C.: Transportation Review Board.

Ong, G. P.; Fwa, T. F. (2007) Wet-pavement hydroplaning risk and skid resistance: modeling. ASCE Journal of transportation Engineering, v. 133, n. 10.

Ong, G. P.; Fwa, T. F. (2008) Modeling and analysis of truck hydroplaning on highways. Transportation Research Record, Washington, D.C., n. 2068, p. 99-108.

Ross, N. F.; Russam, K. (1968) The depth of rain water on road surfaces. London: Ministry of Transport.

Sheridan, L. (2014) Aquaplaning: an investigation of surface flow calculation. Darling Heights: University of South Queensland.

Subramanian, R.; Thiriez, K. (2001) Tire Pressure Special Study Tread Depth Analysis. National Center for Statistics and Analysis of the National Highway Traffic Safety Administration. Washington, D.C.

Transportation Research Board. (2010) Highway Capacity Manual. Washington, D.C: [s.n.]. 